PROCEEDINGS OF THE

AMERICAN MATHEMATICAL SOCIETY

Volume 137, Number 7, July 2009, Pages 2243-2249

S 0002-9939(09)09787-1

Article electronically published on January 28, 2009

\title{
LÉVY CONSTANTS OF TRANSCENDENTAL NUMBERS
}

\author{
CHRISTOPH BAXA
}

(Communicated by Ken Ono)

\begin{abstract}
We prove that every $\gamma \geq \log \frac{1+\sqrt{5}}{2}$ is the Lévy constant of a transcendental number; i.e., there exists a transcendental number $\alpha$ such that $\gamma=$ $\lim _{m \rightarrow \infty} \frac{1}{m} \log q_{m}(\alpha)$, where $q_{m}(\alpha)$ denotes the denominator of the $m$ th convergent of $\alpha$.
\end{abstract}

\section{INTRODUCTION}

An irrational number is said to have a Lévy constant $\beta(\alpha)$ if the limit $\lim _{m \rightarrow \infty} \frac{1}{m} \log q_{m}(\alpha)$ exists and its value is $\beta(\alpha)$. Here $q_{m}(\alpha)$ denotes the denominator of the $m$ th convergent of the regular continued fraction expansion of $\alpha$. Classic results of A.Ya. Khintchine 10 and P. Lévy 11 say that almost every real number has a Lévy constant and its value is $\beta(\alpha)=\pi^{2} /(12 \log 2)$ for almost all $\alpha$.

Lévy constants satisfy $\beta(\alpha) \geq \log ((1+\sqrt{5}) / 2)$. This follows from $[0, \underbrace{1, \ldots, 1}_{m \text { times }}]=$ $F_{m-1} / F_{m}$ (where $F_{m}$ denotes the $m$ th Fibonacci number) and therefore

$$
q_{m}(\alpha) \geq F_{m} \sim \frac{1}{\sqrt{5}}\left(\frac{1+\sqrt{5}}{2}\right)^{m+1} \text { as } m \rightarrow \infty .
$$

H. Jager and P. Liardet 9] proved that every quadratic irrationality has a Lévy constant. C. Faivre [5] showed that every possible value is attained; i.e., for all $\gamma \geq \log ((1+\sqrt{5}) / 2)$ there is an irrational $\alpha$ such that $\beta(\alpha)=\gamma$. E.P. Golubeva [6], 7], 8] studied Lévy constants of quadratic irrationalities and their connections with real quadratic fields and binary quadratic forms. In a recent paper, J. Wu 16] proved that the Lévy constants of quadratic irrationalities are dense in $[\log ((1+\sqrt{5}) / 2),+\infty)$. This implies trivially that the set $\{\beta(\alpha) \mid \alpha$ is algebraic, $\alpha \notin \mathbb{Q}, \beta(\alpha)$ exists $\}$ is dense in $[\log ((1+\sqrt{5}) / 2),+\infty)$. It is the purpose of the present paper to prove the following complementary result:

Theorem 1. (i) For every $\gamma \geq \log ((1+\sqrt{5}) / 2)$ there exist non-denumerably many, pairwise not equivalent transcendental $\alpha$ such that $\beta(\alpha)=\gamma$.

(ii) For every $\gamma \geq \log ((1+\sqrt{5}) / 2)$ there exist non-denumerably many, pairwise not equivalent $U_{2}$-numbers $\alpha$ such that $\beta(\alpha)=\gamma$.

Received by the editors August 26, 2008, and, in revised form, September 29, 2008.

2000 Mathematics Subject Classification. Primary 11K50, 11J81.

(C)2009 American Mathematical Society 
Remarks. 1) Two numbers $\alpha_{1}, \alpha_{2}$ are called equivalent if their continued fraction expansions have shapes $\alpha_{1}=\left[b_{0}, b_{1}, \ldots, b_{k}, a_{1}, a_{2}, a_{3}, \ldots\right]$ and $\alpha_{2}=\left[c_{0}, c_{1}, \ldots, c_{\ell}, a_{1}\right.$, $\left.a_{2}, a_{3}, \ldots\right]$. It is easy to see that equivalent numbers have the same Lévy constant (if one of them has one).

2) Obviously part (ii) of the theorem implies part (i). Nevertheless, we will give separate proofs of both parts as it is instructive to see how certain parameters have to be changed when asking for a $U_{2}$-number instead of a transcendental number.

\section{Continued fractions with Prescribed LÉvy COnStant}

An important technical tool in our proof will be continuants. Let $a_{1}, \ldots, a_{n}$ be positive integers. The continuant $K_{n}\left(a_{1}, \ldots, a_{n}\right)$ is defined as the determinant

$$
K_{n}\left(a_{1}, \ldots, a_{n}\right)=\left|\begin{array}{ccccc}
a_{1} & 1 & & & \\
-1 & a_{2} & 1 & & \\
& -1 & a_{3} & 1 & \\
& & \ddots & \ddots & \ddots \\
& & & -1 & a_{n}
\end{array}\right| .
$$

In addition we set $K_{0}:=1$ and $K_{-1}:=0$.

Lemma 2. (i) $K_{m}\left(a_{1}, \ldots, a_{m}\right)=K_{m}\left(a_{m}, \ldots, a_{1}\right)$.

(ii) For $0 \leq m \leq n$ we have

$$
\begin{aligned}
K_{n}\left(a_{1}, \ldots, a_{n}\right)= & K_{m}\left(a_{1}, \ldots, a_{m}\right) K_{n-m}\left(a_{m+1}, \ldots, a_{n}\right) \\
& +K_{m-1}\left(a_{1}, \ldots, a_{m-1}\right) K_{n-m-1}\left(a_{m+2}, \ldots, a_{n}\right) .
\end{aligned}
$$

(iii) If $\alpha=\left[a_{0}, a_{1}, a_{2}, \ldots\right]$, then $q_{n}(\alpha)=K_{n}\left(a_{1}, \ldots, a_{n}\right)$.

Proof. Part (i) is trivial. Proofs of parts (ii) and (iii) can be found, e.g., in O. Perron's classic textbook [13].

We will drop the index and write $K\left(a_{1}, \ldots, a_{n}\right)$ from now on.

Lemma 3. $\log K\left(a_{1}, \ldots, a_{n}, b_{m}, \ldots, b_{1}\right)=\log K\left(a_{1}, \ldots, a_{n}\right)+\log K\left(b_{1}, \ldots, b_{m}\right)+$ $O(1)$ with an absolute implied constant.

Proof. Using Lemma 2 we get

$$
\begin{aligned}
& \log K\left(a_{1}, \ldots, a_{n}, b_{m}, \ldots, b_{1}\right) \\
& =\log \left(K\left(a_{1}, \ldots, a_{n}\right) K\left(b_{1}, \ldots, b_{m}\right)+K\left(a_{1}, \ldots, a_{n-1}\right) K\left(b_{1}, \ldots, b_{m-1}\right)\right) \\
& =\log K\left(a_{1}, \ldots, a_{n}\right)+\log K\left(b_{1}, \ldots, b_{m}\right) \\
& \quad+\log \left(1+\frac{K\left(a_{1}, \ldots, a_{n-1}\right) K\left(b_{1}, \ldots, b_{m-1}\right)}{K\left(a_{1}, \ldots, a_{n}\right) K\left(b_{1}, \ldots, b_{m}\right)}\right),
\end{aligned}
$$

which implies the assertion.

Lemma 4. If $\alpha=[0, \bar{a}]=[0, a, a, a, \ldots]$, then

$$
\log q_{m}(\alpha)=m \log [\bar{a}]+O(1)=m \log \frac{a+\sqrt{a^{2}+4}}{2}+O(1) .
$$


Proof. Using induction on $m$ one sees that

$$
q_{m}(\alpha)=\frac{1}{\sqrt{a^{2}+4}}\left([\bar{a}]^{m+1}-(-[0, \bar{a}])^{m+1}\right)
$$

for all $m \geq-1$, which implies the assertion.

From now on we will use the shorthand notation

$$
w_{a}=\log [\bar{a}]=\log \frac{a+\sqrt{a^{2}+4}}{2} .
$$

Lemma 5. Let $a<b$ be positive integers and $w_{a}<\gamma<w_{b}$. Then there exist nondenumerably many, pairwise not equivalent $\alpha=\left[0, a_{1}, a_{2}, a_{3}, \ldots\right]$ such that $\beta(\alpha)=\gamma$ and $a_{i} \in\{a, b\}$ for all $i \geq 1$.

Proof. Let $x \in(0,1)$ be such that $\gamma=(1-x) w_{a}+x w_{b}$ and let $\left(\sigma_{k}\right)_{k \geq 1}$ be an increasing sequence of positive integers satisfying $\min \left\{\sigma_{1} x, \sigma_{1}(1-x)\right\} \geq 1, \sigma_{k} \ll k$ as $k \rightarrow \infty$ and $\lim _{k \rightarrow \infty} \sigma_{k}=+\infty$. These conditions imply $\lim _{k \rightarrow \infty} k /\left(\sigma_{1}+\cdots+\sigma_{k}\right)=0$. Set

$$
\alpha=\left[0, \bar{a}^{\left\lfloor(1-x) \sigma_{1}\right\rfloor}, \bar{b}^{\left\lfloor x \sigma_{1}\right\rfloor}, \bar{a}^{\left\lfloor(1-x) \sigma_{2}\right\rfloor}, \bar{b}^{\left\lfloor x \sigma_{2}\right\rfloor}, \ldots\right],
$$

where the notation $\bar{a}^{\tau}$ means that the partial quotient $a$ is repeated $\tau$ times. Let

$$
\sum_{i=1}^{k}\left(\left\lfloor(1-x) \sigma_{i}\right\rfloor+\left\lfloor x \sigma_{i}\right\rfloor\right)<m \leq \sum_{i=1}^{k+1}\left(\left\lfloor(1-x) \sigma_{i}\right\rfloor+\left\lfloor x \sigma_{i}\right\rfloor\right) .
$$

Then

$$
\left(a_{1}, \ldots, a_{m}\right)=\left(\bar{a}^{\left\lfloor(1-x) \sigma_{1}\right\rfloor}, \bar{b}^{\left\lfloor x \sigma_{1}\right\rfloor}, \ldots, \bar{a}^{\left\lfloor(1-x) \sigma_{k}\right\rfloor}, \bar{b}^{\left\lfloor x \sigma_{k}\right\rfloor}, \bar{a}^{\tau_{a}}, \bar{b}^{\tau_{b}}\right)
$$

for some $\tau_{a}, \tau_{b}$ satisfying either $1 \leq \tau_{a} \leq\left\lfloor(1-x) \sigma_{k+1}\right\rfloor$ and $\tau_{b}=0$ or $\tau_{a}=$ $\left\lfloor(1-x) \sigma_{k+1}\right\rfloor$ and $1 \leq \tau_{b} \leq\left\lfloor x \sigma_{k+1}\right\rfloor$. Using Lemmata 3 and 4 we get

$$
\begin{aligned}
\log q_{m}(\alpha)= & \log K\left(\bar{a}^{\left\lfloor(1-x) \sigma_{1}\right\rfloor}, \bar{b}^{\left\lfloor x \sigma_{1}\right\rfloor}, \ldots, \bar{a}^{\left\lfloor(1-x) \sigma_{k}\right\rfloor}, \bar{b}^{\left\lfloor x \sigma_{k}\right\rfloor}, \bar{a}^{\tau_{a}}, \bar{b}^{\tau_{b}}\right) \\
= & \sum_{i=1}^{k}\left(\log K\left(\bar{a}^{\left\lfloor(1-x) \sigma_{i}\right\rfloor}\right)+\log K\left(\bar{b}^{\left\lfloor x \sigma_{i}\right\rfloor}\right)\right) \\
& +\log K\left(\bar{a}^{\tau_{a}}\right)+\log K\left(\bar{b}^{\tau_{b}}\right)+O(k) \\
= & \sum_{i=1}^{k}\left(\left\lfloor(1-x) \sigma_{i}\right\rfloor w_{a}+\left\lfloor x \sigma_{i}\right\rfloor w_{b}\right)+\tau_{a} w_{a}+\tau_{b} w_{b}+O(k) \\
= & (1-x)\left(\sigma_{1}+\cdots+\sigma_{k}\right) w_{a}+x\left(\sigma_{1}+\cdots+\sigma_{k}\right) w_{b}+O(k) .
\end{aligned}
$$

Analogously we have $m=\sigma_{1}+\cdots+\sigma_{k}+O(k)$, which, together with (1), implies the assertion.

\section{Transcendence CRITERIA}

The transcendence criteria we use follow ideas that originated with E. Maillet 12 and A. Baker 2, 3]. Recent improvements of their work can be found in papers by J.L. Davison [4] and B. Adamczewski and Y. Bugeaud [1]. 
Theorem 6 (W.M. Schmidt, [14; see also [15]). Let $\alpha \in \mathbb{R}$ be algebraic but neither rational nor a quadratic irrationality and $\delta>0$. Then there exist only finitely many $\beta \in \mathbb{R}$ which are rational or quadratic irrationalities such that $|\alpha-\beta|<H(\beta)^{-3-\delta}$.

Remark. Here $H$ denotes the classic absolute height; i.e., if $p(X)=\sum_{i=0}^{m} a_{i} X^{i} \in$ $\mathbb{Z}[X] \backslash\{0\}$ with $\operatorname{gcd}\left(a_{0}, \ldots, a_{m}\right)=1, p(\beta)=0$ and $\operatorname{deg} p$ is minimal with this property, then $H(\beta)=\max _{0 \leq i \leq m}\left|a_{i}\right|$.

Corollary 7. Let $\alpha \in \mathbb{R}$ have a quasiperiodic but not periodic continued fraction expansion

$$
\alpha=\left[0, a_{1}, \ldots, a_{\nu_{1}-1},{\overline{a_{\nu_{1}}, \ldots, a_{\nu_{1}+k_{1}-1}}}^{\lambda_{1}},{\overline{a_{\nu_{2}}, \ldots, a_{\nu_{2}+k_{2}-1}}}^{\lambda_{2}}, \ldots\right] .
$$

Here $\overline{a_{\nu}, \ldots, a_{\nu+k}} \lambda$ indicates that the partial quotients $a_{\nu}, \ldots, a_{\nu+k}$ should be repeated $\lambda$ times, i.e., $\nu_{n}=\nu_{1}+\sum_{i=1}^{n-1} \lambda_{i} k_{i}$. If $\alpha$ is algebraic, then $\varlimsup_{i \rightarrow \infty} q_{\nu_{i+1}-1} q_{\nu_{i}+k_{i}-1}^{-3-\delta}$ $<+\infty$ for every $\delta>0$.

Proof. For $i \geq 1$ we define the quadratic irrationality

$$
\beta_{i}=\left[0, a_{1}, \ldots, a_{\nu_{1}-1}, \overline{a_{\nu_{1}}, \ldots, a_{\nu_{1}+k_{1}-1}} \lambda_{1}, \ldots, \overline{a_{\nu_{i-1}}, \ldots, \overline{a_{\nu_{i-1}+k_{i-1}-1}} \lambda_{i-1}},\right.
$$

For $k \leq \nu_{i+1}-1$ we have $a_{k}(\alpha)=a_{k}\left(\beta_{i}\right)$ and we may write $p_{k} / q_{k}$ for $p_{k}(\alpha) / q_{k}(\alpha)=$ $p_{k}\left(\beta_{i}\right) / q_{k}\left(\beta_{i}\right)$. Now $L_{i} \beta_{i}^{2}+M_{i} \beta_{i}+N_{i}=0$ with

$$
\begin{aligned}
L_{i} & =q_{\nu_{i}-2} q_{\nu_{i}+k_{i}-1}-q_{\nu_{i}-1} q_{\nu_{i}+k_{i}-2} \\
M_{i} & =q_{\nu_{i}-1} p_{\nu_{i}+k_{i}-2}+p_{\nu_{i}-1} q_{\nu_{i}+k_{i}-2}-p_{\nu_{i}-2} q_{\nu_{i}+k_{i}-1}-q_{\nu_{i}-2} p_{\nu_{i}+k_{i}-1} \\
N_{i} & =p_{\nu_{i}-2} p_{\nu_{i}+k_{i}-1}-p_{\nu_{i}-1} p_{\nu_{i}+k_{i}-2}
\end{aligned}
$$

and therefore $H\left(\beta_{i}\right) \leq \max \left\{\left|L_{i}\right|,\left|M_{i}\right|,\left|N_{i}\right|\right\}<2 q_{\nu_{i}+k_{i}-1}^{2}$. Theorem 6 implies

$$
q_{\nu_{i+1}-1}^{-2}>\left|\alpha-\beta_{i}\right|>C(\alpha, \delta) H\left(\beta_{i}\right)^{-3-\delta}>C(\alpha, \delta) 2^{-3-\delta} q_{\nu_{i}+k_{i}-1}^{-6-2 \delta}
$$

for a certain $C(\alpha, \delta)>0$. The corollary follows immediately.

Lemma 8. Let $a<b$ be positive integers and $w_{a}<\gamma<w_{b}$. Then there exist nondenumerably many, pairwise not equivalent transcendental $\alpha=\left[0, a_{1}, a_{2}, a_{3}, \ldots\right]$ such that $\beta(\alpha)=\gamma$ and $a_{i} \in\{a, b\}$ for all $i \geq 1$.

Proof. Let $x$ and $\left(\sigma_{k}\right)_{k \geq 1}$ be as in the proof of Lemma 5. Set

$$
\alpha=\left[0,{\overline{\bar{a}}\left\lfloor(1-x) \sigma_{1}\right\rfloor}_{\bar{b}^{\left\lfloor x \sigma_{1}\right\rfloor}}^{\lambda_{1}},{\overline{\bar{a}^{\left\lfloor(1-x) \sigma_{2}\right\rfloor}, \bar{b}^{\left\lfloor x \sigma_{2}\right\rfloor}}}^{\lambda_{2}}, \ldots\right] .
$$

Then $\beta(\alpha)=\gamma$ by Lemma 5 . Using the notation of Corollary 7 we have

(2) $k_{n}=\left\lfloor(1-x) \sigma_{n}\right\rfloor+\left\lfloor x \sigma_{n}\right\rfloor$ and $\nu_{n}-1=\sum_{i=1}^{n-1} \lambda_{i}\left(\left\lfloor(1-x) \sigma_{i}\right\rfloor+\left\lfloor x \sigma_{i}\right\rfloor\right)$ for all $n \geq 1$.

Employing that $[\bar{a}]^{m-1} \leq q_{m}(\alpha) \leq[\bar{b}]^{m}$ for all $m \geq 0$ we get

$$
\log q_{\nu_{n+1}-1}(\alpha) \geq\left(-1+\sum_{i=1}^{n} \lambda_{i}\left(\left\lfloor(1-x) \sigma_{i}\right\rfloor+\left\lfloor x \sigma_{i}\right\rfloor\right)\right) w_{a}
$$


and

$\log q_{\nu_{n}+k_{n}-1}^{-7 / 2}(\alpha) \geq-\frac{7}{2} \frac{w_{b}}{w_{a}}\left(\sum_{i=1}^{n-1} \lambda_{i}\left(\left\lfloor(1-x) \sigma_{i}\right\rfloor+\left\lfloor x \sigma_{i}\right\rfloor\right)+\left\lfloor(1-x) \sigma_{n}\right\rfloor+\left\lfloor x \sigma_{n}\right\rfloor\right) w_{a}$.

Because of Corollary 7, $\alpha$ will be transcendental if the sequence $\left(\lambda_{n}\right)_{n \geq 1}$ is chosen such that

$$
\begin{array}{r}
\varlimsup_{n \rightarrow \infty}\left(\left(\lambda_{n}-\frac{7}{2} \frac{w_{b}}{w_{a}}\right)\left(\left\lfloor(1-x) \sigma_{n}\right\rfloor+\left\lfloor x \sigma_{n}\right\rfloor\right)-\left(\frac{7}{2} \frac{w_{b}}{w_{a}}-1\right) \sum_{i=1}^{n-1} \lambda_{i}\left(\left\lfloor(1-x) \sigma_{i}\right\rfloor+\left\lfloor x \sigma_{i}\right\rfloor\right)\right) \\
=+\infty .
\end{array}
$$

As the sequence $\left(\lambda_{n}\right)_{n>1}$ can be chosen in non-denumerably many different ways, the construction yields non-denumerably many, pairwise not equivalent $\alpha$ with $\beta(\alpha)=\gamma$.

Lemma 9. Keeping the notation of Corollary 7 we have

$$
0<\left|L_{n} \alpha^{2}+M_{n} \alpha+N_{n}\right|<8 q_{\nu_{n}+k_{n}-1}^{4} q_{\nu_{n+1}-1}^{-2} .
$$

Proof. Let $\bar{\beta}_{i}$ denote the conjugate of $\beta_{i}$. If $\left|\bar{\beta}_{i}\right| \geq 1$, it follows from $L_{i} \bar{\beta}_{i}^{2}+M_{i} \bar{\beta}_{i}+$ $N_{i}=0$ that

$$
\left|\bar{\beta}_{i}\right|^{2} \leq\left|L_{i} \bar{\beta}_{i}^{2}\right|=\left|M_{i} \bar{\beta}_{i}+N_{i}\right|<2 q_{\nu_{i}+k_{i}-1}^{2}\left(\left|\bar{\beta}_{i}\right|+1\right) \leq 4 q_{\nu_{i}+k_{i}-1}^{2}\left|\bar{\beta}_{i}\right|
$$

and therefore $\left|\bar{\beta}_{i}\right|<4 q_{\nu_{i}+k_{i}-1}^{2}$, which remains true even if $\left|\bar{\beta}_{i}\right|<1$. This implies

$$
\left|\alpha-\bar{\beta}_{i}\right| \leq 1+\left|\bar{\beta}_{i}\right|<1+4 q_{\nu_{i}+k_{i}-1}^{2}<8 q_{\nu_{i}+k_{i}-1}^{2}
$$

and thus

$$
\begin{aligned}
\left|L_{i} \alpha^{2}+M_{i} \alpha+N_{i}\right| & =\left|L_{i}\right| \cdot\left|\alpha-\beta_{i}\right| \cdot\left|\alpha-\bar{\beta}_{i}\right| \\
& <q_{\nu_{i}+k_{i}-1}^{2} \cdot q_{\nu_{i+1}-1}^{-2} \cdot 8 q_{\nu_{i}+k_{i}-1}^{2}=8 q_{\nu_{i}+k_{i}-1}^{4} q_{\nu_{i+1}-1}^{-2}
\end{aligned}
$$

Lemma 10. Let $a<b$ be positive integers and $w_{a}<\gamma<w_{b}$. Then there exist non-denumerably many, pairwise not equivalent $U_{2}$-numbers $\alpha=\left[0, a_{1}, a_{2}, a_{3}, \ldots\right]$ such that $\beta(\alpha)=\gamma$ and $a_{i} \in\{a, b\}$ for all $i \geq 1$.

Proof. Let $x$ and $\left(\sigma_{k}\right)_{k \geq 1}$ be as in the proof of Lemma 5 and $\alpha$ as in the proof of Lemma 8 . Then $\beta(\alpha)=\gamma$ by Lemma 5 and (2) holds. We have

$$
H\left(L_{n} X^{2}+M_{n} X+N_{n}\right)=\max \left\{\left|L_{n}\right|,\left|M_{n}\right|,\left|N_{n}\right|\right\}<2 q_{\nu_{n}+k_{n}-1}^{2} \leq 2[\bar{b}]^{2\left(\nu_{n}+k_{n}-1\right)},
$$

where $H$ denotes the height of a polynomial. Using Lemma 9 we can estimate

$$
\begin{aligned}
0 & <\left|L_{n} \alpha^{2}+M_{n} \alpha+N_{n}\right|<8 q_{\nu_{n}+k_{n}-1}^{4} q_{\nu_{n+1}-1}^{-2} \leq 8[\bar{b}]^{4\left(\nu_{n}+k_{n}-1\right)}[\bar{a}]^{-2\left(\nu_{n+1}-2\right)} \\
& =[\bar{b}]^{-\left(2\left(\nu_{n+1}-2\right) w_{a}-4\left(\nu_{n}+k_{n}-1\right) w_{b}-3 \log 2\right) / w_{b}}=\left(2[\bar{b}]^{2\left(\nu_{n}+k_{n}-1\right)}\right)^{-\Psi_{n}}
\end{aligned}
$$

with

$$
\Psi_{n}=\frac{2\left(\nu_{n+1}-2\right) w_{a}-4\left(\nu_{n}+k_{n}-1\right) w_{b}-3 \log 2}{2\left(\nu_{n}+k_{n}-1\right) w_{b}+\log 2} .
$$

Replacing $\nu_{n}$ and $k_{n}$ by the expressions given in (2) we see that $\varlimsup_{n \rightarrow \infty} \Psi_{n}=+\infty$ can be achieved by letting the sequence $\left(\lambda_{n}\right)_{n \geq 1}$ grow sufficiently fast, which proves the assertion. 
Remark. As $\left(\log \frac{1+\sqrt{5}}{2},+\infty\right)=\bigcup_{n=1}^{\infty}\left(w_{n}, w_{n+2}\right)$, the assertions of Theorem 1 follow from Lemma 8 and Lemma 10 respectively, at least for every $\gamma>\log \frac{1+\sqrt{5}}{2}$. In the remainder we will briefly indicate how one can take care of the remaining value $w_{1}$.

Lemma 11. If $\alpha=\left[a_{0}, a_{1}, a_{2}, \ldots\right]$ has a Lévy constant, then

$$
\beta(\alpha)=\lim _{m \rightarrow \infty} \frac{1}{m} \sum_{i=1}^{m} \log \left[a_{i}, a_{i+1}, a_{i+2}, \ldots\right] .
$$

Proof. Using well-known identities from the theory of regular continued fractions one gets

$$
\prod_{i=1}^{m+1}\left[a_{i}, a_{i+1}, \ldots\right]=\left|q_{m} \alpha-p_{m}\right|^{-1}=q_{m} \cdot\left[a_{m+1}, a_{m+2}, \ldots\right]+q_{m-1}
$$

(where $p_{m} / q_{m}$ denotes the $m$ th convergent), which implies

$$
\begin{aligned}
\sum_{i=1}^{m+1} \log \left[a_{i}, a_{i+1}, \ldots\right] & =\log q_{m}+\log \left(\left[a_{m+1}, a_{m+2}, \ldots\right]+\frac{q_{m-1}}{q_{m}}\right) \\
& =\log q_{m}+\log \left(\left[a_{m+1}, a_{m+2}, \ldots\right]+\left[0, a_{m}, \ldots, a_{1}\right]\right) \\
& =\log q_{m}+\log \left[a_{m+1}, a_{m+2}, \ldots\right]+O(1) .
\end{aligned}
$$

Lemma 12. Let $\alpha=\left[a_{0}, a_{1}, a_{2}, \ldots\right], \alpha^{\prime}=\left[b_{0}, b_{1}, b_{2}, \ldots\right]$ and let $t(m)$ denote the number of $i \in\{1, \ldots, m\}$ with $a_{i} \neq b_{i}$. If $\alpha$ has a Lévy constant, $b_{i}=a_{i}+O(1)$ as $i \rightarrow \infty$ and $t(m)=o(m)$ as $m \rightarrow \infty$, then $\beta\left(\alpha^{\prime}\right)=\beta(\alpha)$.

Proof. We have

$$
\begin{aligned}
\sum_{i=1}^{m} \log \left[b_{i}, b_{i+1}, \ldots\right] & =\sum_{i=1}^{m} \log \left[a_{i}, a_{i+1}, \ldots\right]+\sum_{i=1}^{m}\left(\log \left[b_{i}, b_{i+1}, \ldots\right]-\log \left[a_{i}, a_{i+1}, \ldots\right]\right) \\
& =\sum_{i=1}^{m} \log \left[a_{i}, a_{i+1}, \ldots\right]+O(t(m))=\sum_{i=1}^{m} \log \left[a_{i}, a_{i+1}, \ldots\right]+o(m),
\end{aligned}
$$

which together with Lemma 11 yields the assertion.

Lemma 13. Let $a<b$ be positive integers and $\alpha=\left[0, \bar{a}^{\lambda_{1}}, \bar{b}^{\lambda_{2}}, \bar{a}^{\lambda_{3}}, \bar{b}^{\lambda_{4}}, \ldots\right]$.

(i) If

$$
\varlimsup_{n \rightarrow \infty}\left(\lambda_{1}+\cdots+\lambda_{n}-\frac{7}{2} \frac{w_{b}}{w_{a}}\left(\lambda_{1}+\cdots+\lambda_{n-1}\right)\right)=+\infty,
$$

then $\alpha$ is transcendental.

(ii) If $\varlimsup_{n \rightarrow \infty} \lambda_{n} /\left(\lambda_{1}+\cdots+\lambda_{n-1}\right)=+\infty$, then $\alpha$ is a $U_{2}$-number.

Proof. This can be proved along the same lines as Lemmata 8 and 10.

Lemma 14. Let a be a positive integer.

(i) There exist non-denumerably many pairwise not equivalent transcendental $\alpha$ such that $\beta(\alpha)=w_{a}$.

(ii) There exist non-denumerably many pairwise not equivalent $U_{2}$-numbers $\alpha$ such that $\beta(\alpha)=w_{a}$. 
Proof. Set $\alpha=\left[0, \bar{a}^{\lambda_{1}}, a+1, \bar{a}^{\lambda_{3}}, a+1, \bar{a}^{\lambda_{5}}, \ldots\right]$. By letting the sequence $\left(\lambda_{2 n+1}\right)_{n \geq 0}$ grow fast enough we can guarantee that the assumptions of both Lemma 12 and Lemma 13 are satisfied.

\section{REFERENCES}

1. B. Adamczewski and Y. Bugeaud, On the Maillet-Baker continued fractions, J. Reine Angew. Math. 606 (2007), 105-121. MR2337643 (2008d:11073)

2. A. Baker, Continued fractions of transcendental numbers, Mathematika 9 (1962), 1-8. MR 0144853 (26:2394)

3. A. Baker, On Mahler's classification of transcendental numbers, Acta Math. 111 (1964), 97-120. MR0157943 (28:1171)

4. J. L. Davison, Quasi-periodic continued fractions, J. Number Theory 127 (2007), 272-282. MR 2362436

5. C. Faivre, The Lévy constant of an irrational number, Acta Math. Hungar. 74 (1997), 57-61. MR.1428047 (97j:11036)

6. E. P. Golubeva, Estimates of the Lévy constant for $\sqrt{p}$ and a class number one criterion for Q $(\sqrt{p})$, J. Math. Sci. (N.Y.) 95 (1999), 2185-2191. MR1691280(2000d:11129)

7. E. P. Golubeva, The spectrum of Lévy constants for quadratic irrationalities, J. Math. Sci. (N.Y.) 110 (2002), 3040-3047. MR1756334 (2001b:11065)

8. E. P. Golubeva, The spectrum of Lévy constants for quadratic irrationalities and class numbers of real quadratic fields, J. Math. Sci. (N.Y.) 118 (2003), 4740-4752. MR1850361 (2002k:11199)

9. H. Jager and P. Liardet, Distributions arithmétiques des dénominateurs de convergents de fractions continues, Indag. Math. 50 (1988), 181-197. MR952514 (89i:11085)

10. A. Ya. Khintchine, Zur metrischen Kettenbruchtheorie, Compositio Math. 3 (1936), 276-285. MR 1556944

11. P. Lévy, Sur le dévelopment en fraction continue d'un nombre choisi au hasard, Comp. Math. 3 (1936), 286-303.

12. E. Maillet, Introduction à la théorie des nombres transcendants et des propriétés arithmétiques des fonctions, Gauthier-Villars, Paris, 1906.

13. O. Perron, Die Lehre von den Kettenbrüchen, Band 1. Elementare Kettenbrüche, Teubner, Stuttgart, 1977. MR0064172(16:239e)

14. W.M. Schmidt, On simultaneous approximations of two algebraic numbers by rationals, Acta Math. 119 (1967), 27-50. MR0223309 (36:6357)

15. W.M. Schmidt, Diophantine Approximation, Lecture Notes in Math. 785, Springer, Berlin, 1980. MR $568710(81 \mathrm{j}: 10038)$

16. J. Wu, On the Lévy constants for quadratic irrationals, Proc. Amer. Math. Soc. 134 (2006), 1631-1634. MR2204273(2007a:11112)

Department of Mathematics, University of Vienna, Nordbergstrasse 15, A-1090, Wien, Austria

E-mail address: christoph.baxa@univie.ac.at 